\title{
Differential blood lipid-lowering effects of alkylsulfonated chitosan of different molecular weights in Syrian hamsters in vivo
}

\author{
CHIH-CHUNG WU ${ }^{1}$, SHAN-YING LIN ${ }^{2}$, CHI-TSAI CHEN ${ }^{3}$, YUEH-PING CHANG ${ }^{4}$, YUN-SHSAN HUANG ${ }^{1}$, \\ CHONG-KUEI LII ${ }^{5}$, CHIEN-CHIH YU ${ }^{6}$, SHU-LING HSIEH ${ }^{7 *}$ and JING-GUNG CHUNG ${ }^{8,9 *}$ \\ ${ }^{1}$ Department of Nutrition and Health Sciences, Chang Jung Christian University, Tainan 71101; \\ ${ }^{2}$ Innovation Development Center, Taiwan Hopax Chemicals MFG. Co., Ltd., Kaohsiung 83162; \\ ${ }^{3}$ Department of Restaurant and Hospitality Management, Chung Hwa University of Medical Technology, Tainan 71703; \\ ${ }^{4}$ Department of Nutrition and Health Science, Fooyin University, Kaohsiung 83102; ${ }^{5}$ Department of Nutrition, \\ China Medical University, Taichung 40402; ${ }^{6}$ School of Pharmacy, China Medical University, Taichung 40402; \\ ${ }^{7}$ Department of Seafood Sciences, National Kaohsiung Marine University, Kaohsiung 81143; \\ ${ }^{8}$ Department of Biological Science and Technology, China Medical University, Taichung 40402; \\ ${ }^{9}$ Department of Biotechnology, Asia University, Taichung 41354, Taiwan, R.O.C.
}

Received August 2,2011; Accepted December 9, 2011

DOI: $10.3892 / \mathrm{mmr} .2011 .705$

\begin{abstract}
This study investigated the effects of alkylsulfonated chitosan of different molecular weights on intestinal lipid absorption, blood lipid profiles and circulating adhesion molecules. Syrian hamsters were fed an AIN-93G-based high-fat diet (HFD) and were orally administered 5 or $10 \mathrm{mg} / \mathrm{kg} \mathrm{BW}$ of oligomer (6 kDa) chitosan (OC), low-molecular-weight (70 kDa) chitosan (LMC) or high-molecular-weight $(200 \mathrm{kDa})$ chitosan (HMC) four times per week for 12 weeks. Animals receiving $2.5 \mathrm{mg} / \mathrm{kg}$ BW lovastatin (LOVA) served as a positive control. The blood lipid profiles of these control animals revealed that all chitosans and LOVA significantly decreased total triglyceride, total cholesterol, low-density lipoprotein (LDL)-cholesterol and very-low-density lipoprotein (VLDL)-cholesterol levels in a dose-dependent manner compared to the HFD-fed controls $(\mathrm{P}<0.05)$. The blood lipid lowering effectiveness of the three chitosans followed the order of LMC $>\mathrm{OC}>\mathrm{HMC}$. Hamsters receiving 5 and $10 \mathrm{mg} / \mathrm{kg} \mathrm{LMC}(\mathrm{P}<0.05)$ exhibited an increase in fecal fat content. Immunoblot assay revealed that acyl-coen-
\end{abstract}

Correspondence to: Dr Jing-Gung Chung, Department of Biological Science and Technology, China Medical University. No 91, Hsueh-Shih Road, Taichung 40402, Taiwan, R.O.C.

E-mail: jgchung@mail.cmu.edu.tw

Dr Shu-Ling Hsieh, Department of Seafood Sciences, National Kaohsiung Marine University, No. 42 Haijhuan Road, Nanzih District, Kaohsiung 81157, Taiwan, R.O.C.

E-mail: slhsieh@ntu.edu.tw

*Contributed equally

Key words: acyl-coenzyme A:cholesterol acyltransferase-2, blood lipids, chitosan, hamsters zyme A:cholesterol acyltransferase-2 (ACAT-2) expression was suppressed in all chitosan-fed animals compared to the HFD-fed controls $(\mathrm{P}<0.05)$. These results suggest that chitosan effectively decreases blood lipid content, and its effectiveness depends on the molecular size of chitosan. The hypolipidemic effect of chitosan is partly attributed to its suppression of intestinal lipid absorption and hepatic ACAT-2 expression.

\section{Introduction}

Chitosan is a biopolymer of glucosamine produced by the deacetylation of chitin, which is an aminopolysaccharide found in the exoskeleton of arthropods and the cell wall of fungi. Chitosan is chemically similar to cellulose. Previous studies have shown that chitosan and its oligomers display a variety of biological activities, including antitumor, hypoglycemic, hypolipidemic, antibacterial and eight control effects (1-5). The effectiveness of chitosan in lowering blood lipids and in body weight control allows it to decrease the risk of cardiovascular disease (6-8).

Chitosan is generated from chitin. The extent of hydrolysis and deacetylation produces chitosan with various molecular weights and viscosity. The oligo-form of chitosan, which is generated by the degradation of the chitosan polymer chain, shows better water solubility and antitumor effects than the poly-form of chatoyant (9). Molecular weight and viscosity are critical factors responsible for the hypolipidemic effect of chatoyant $(3,6,10)$. The molecular weight of chitosan ranging from 20 to $2000 \mathrm{kDa}$ displays better potency for lowering blood triglycerides and cholesterol $(6,11,12)$. Establishing the molecular weight and chemical characteristics of chitosan with optimal biological activity remains an elusive target.

Chitosan is insoluble in water and organic solvents. To increase the application of chitosan in the food and medical industry, alkylsulfonation produces a novel modified chatoyant $(13,14)$. This type of alkylsulfonated chitosan 
retains the structure and characteristics of the natural polymer and is a water soluble and anionic polymer as a result of the introduction of a hydrophilic acid functional group.

3-Hydroxy-3-methylglutaryl coenzyme A (HMG CoA) reductase catalyzes the conversion of $\mathrm{HMG} \mathrm{CoA}$ to the mevalonate pathway, and is the rate-limiting enzyme of cholesterol biosynthesis $(15,16)$. The inhibition of HMG CoA reductase activity suppresses hepatic cholesterol formation, which in turn decreases blood cholesterol levels. The hypocholesterolemic effects of statins and phytochemicals, including garlic organosulfur compounds and soy isoflavones, have been attributed to their potent inhibition of this enzyme activity (16-19). Acyl-coenzyme A:cholesterol acyltransferase (ACAT) is another key enzyme that regulaes plasma cholesterol. ACAT catalyzes cholesteryl esters from cholesterol and fatty acyl$\mathrm{CoA}$, and is responsible for intestinal cholesterol absorption, hepatic repackaging of very-low density lipoprotein (VLDL), cholesterol storage for steroid hormone biosynthesis $(20,21)$ and the formation of macrophage-derived foam cells in atherosclerotic lesions (22). Bavachin and isobavachalcone inhibition of ACAT activity partly explains the blood cholesterol-lowering effect of Psoralea corylifolia (23). In addition to interfering with lipid absorption in the gut, the role of chitosan in modulating $\mathrm{HMG}-\mathrm{CoA}$ reductase and ACTA activity and expression in hamster livers remains unclear.

The experiments in this study assessed three alkylsulfonated chitosans with different molecular weights, i.e. 6, 70, and $200 \mathrm{kDa}$, for their hypolipidemic activity. Blood triglycerides and cholesterol, fecal fat content and hepatic HMG-CoA reductase and ACAT-2 expression were examined in Syrian hamsters.

\section{Materials and methods}

Materials. Alkylsulfonated chitosan was purchased from Taiwan Hopax Chems MFG Co., Ltd. (Kaohsiung, Taiwan). The oligomer chitosan [molecular weight (MW) $6 \mathrm{kDa}$ and low- and high-molecular-weight polymer chitosan (MW 70 and $200 \mathrm{kDa}$, respectively) were the experimental materials used in this study. Lovastatin was purchased from Calbiochem Ltd. (San Diego, CA, USA). The anti-HMG CoA reductase, ACAT-2, intracellular adhesion molecule 1 (ICAM-1) or vascular cell adhesion molecule 1 (VCAM-1), and intracellular adhesion molecule 1 (ICAM-1) or vascular cell adhesion molecule 1 (VCAM-1) monoclonal antibodies were purchased from Santa Cruz Biotechnology, Inc. (Santa Cruz, CA, USA).

Animals and treatments. Hamsters were treated in compliance with the Guide for the Care and Use of Laboratory Animals of the Institute of Laboratory Animal Resources, Commission on Life Sciences, National Research Council, National Academy, Washington D.C., USA and the animal experiments were approved by the Chang Jung Christian University Animal Ethics Committee. Four-week-old male Syrian hamsters were purchased from the National Laboratory Animal Center (Taipei, Taiwan). Following one week of acclimation, hamsters were randomly assigned to each experimental group $(n=6)$ by weight and subjected to a 12-h light cycle. Animals had free access to water and an AIN-93G-based diet (5\% soybean oil, ND) or a high-fat diet
Table I. Composition of the experimental diets.

\begin{tabular}{llc}
\hline Ingredients & ND $(\%)$ & HFD $(\%)$ \\
\hline Corn starch & 41.75 & 26.25 \\
Casein (vitamin-free) & 20 & 20 \\
Maltodextrin & 13.2 & 13.2 \\
Sucrose & 10 & 10 \\
Cellulose & 5 & 5 \\
Soybean oil & 5 & 20 \\
AIN 93G mineral mix & 3.5 & 3.5 \\
AIN 93G vitamin mix & 1 & 1 \\
Cholesterol & 0 & 0.5 \\
L-cystine & 0.3 & 0.3 \\
Choline bitartate & 0.25 & 0.25
\end{tabular}

AIN-93G (ND, normal diet) and a modified AIN-93G diet containing $20 \%$ fat $-0.5 \%$ cholesterol (HFD, high-fat diet) were administered.

(20\% soybean oil plus $0.5 \%$ cholesterol, HFD) (Dyets, Inc., Bethlehem, PA, USA) (Table I). Hamsters fed with the HFD were subdivided into the following treatment groups, and orally administered 5 or $10 \mathrm{mg}$ BW oligomer chitosan (5OC, 10OC), low-molecular-weight chitosan (5LMC, 10 LMC), 5 or $10 \mathrm{mg} / \mathrm{kg}$ BW high-molecular-weight chitosan (5HMC, 10HMC) or $2.5 \mathrm{mg} / \mathrm{kg} \mathrm{BW}$ lovastatin (LOVA, as a positive control). All chitosan products and lovastatin were prepared in phosphate-buffered saline (PBS) and were administered orally 4 times per week for 12 weeks. During the last week, fecal samples from each group were collected in a metabolic cage and were used for total fecal crude fat content examination. Following fasting overnight, hamsters were sacrificed by carbon dioxide euthanasia. Blood was withdrawn using the intracardiac method and serum was prepared for lipid profile analysis. The liver, heart, spleen and kidney were removed and weighed. The largest liver lobes were used for histological examination and the liver remnants were quickly freeze-clamped in liquid nitrogen and stored at $-80^{\circ} \mathrm{C}$ until further analysis. The thoracic aortae were used for ICAM-1 and VCAM-1 determination by immunoblotting assay.

Serum lipid determination. Blood samples were collected and serum was prepared by centrifuging at $1000 \mathrm{x} g$ for $15 \mathrm{~min}$ at $4^{\circ} \mathrm{C}$. Amounts of total cholesterol (TC), triglyceride (TG), low density lipoprotein-cholesterol (LDL-C), high density lipoprotein-cholesterol (HDL-C) and VLDL-C were measured with commercial kits (Randox Laboratories Ltd., San Diego, CA, USA) according to the manufacturer's instructions.

Histological examination. Liver tissues were fixed in $10 \%$ neutral buffered formalin. Sections $(5 \mu \mathrm{m})$ from the paraffin-embedded tissue were stained with hematoxylin and eosin (H\&E). Pathological examination was performed by an expert pathologist in a blinded manner.

Analysis of total fecal crude fat level. Fecal samples were collected in a metabolic cage and were stored at $-20^{\circ} \mathrm{C}$ until 
Table II. Growth characteristics of hamsters administered chitosan.

\begin{tabular}{|c|c|c|c|c|c|c|}
\hline \multirow[t]{2}{*}{ Diet } & \multirow{2}{*}{$\begin{array}{l}\text { Food intake } \\
\text { g/day }\end{array}$} & \multirow{2}{*}{$\begin{array}{l}\text { Body weight gain } \\
\text { g/12 weeks }\end{array}$} & \multirow[t]{2}{*}{ Liver } & Heart & Kidney & Spleen \\
\hline & & & & \multicolumn{3}{|c|}{ Relative to body weight (\%) } \\
\hline ND & $8.2 \pm 2.1$ & $50 \pm 3$ & $5.1 \pm 0.5$ & $0.37 \pm 0.05$ & $0.97 \pm 0.02$ & $0.07 \pm 0.01$ \\
\hline HFD & $8.3 \pm 2.7$ & $48 \pm 3$ & $5.2 \pm 0.6$ & $0.37 \pm 0.05$ & $1.00 \pm 0.02$ & $0.07 \pm 0.01$ \\
\hline $\mathrm{HFD}+5 \mathrm{OC}$ & $8.1 \pm 3.3$ & $51 \pm 3$ & $5.1 \pm 0.6$ & $0.34 \pm 0.05$ & $0.94 \pm 0.10$ & $0.10 \pm 0.03$ \\
\hline HFD+10OC & $6.7 \pm 2.2$ & $51 \pm 3$ & $5.0 \pm 0.6$ & $0.34 \pm 0.04$ & $0.87 \pm 0.06$ & $0.08 \pm 0.03$ \\
\hline HFD+5LMC & $6.8 \pm 3.1$ & $50 \pm 3$ & $5.4 \pm 0.6$ & $0.36 \pm 0.06$ & $0.97 \pm 0.05$ & $0.10 \pm 0.02$ \\
\hline HFD+10LMC & $7.7 \pm 2.3$ & $49 \pm 4$ & $5.3 \pm 0.5$ & $0.33 \pm 0.05$ & $1.00 \pm 0.01$ & $0.08 \pm 0.02$ \\
\hline HFD+5HMC & $7.5 \pm 2.8$ & $50 \pm 5$ & $5.4 \pm 0.7$ & $0.35 \pm 0.06$ & $0.87 \pm 0.06$ & $0.09 \pm 0.03$ \\
\hline HFD+10HMC & $7.8 \pm 2.2$ & $50 \pm 3$ & $5.5 \pm 0.7$ & $0.37 \pm 0.07$ & $0.97 \pm 0.06$ & $0.10 \pm 0.02$ \\
\hline HFD+LOVA & $7.4 \pm 2.7$ & $51 \pm 3$ & $5.1 \pm 0.7$ & $0.33 \pm 0.07$ & $0.90 \pm 0.10$ & $0.09 \pm 0.03$ \\
\hline
\end{tabular}

Hamsters were fed a normal diet (ND) or high-fat diet (HFD), and those fed the HFD were sub-grouped and orally administered $5 \mathrm{or} 10 \mathrm{mg} / \mathrm{kg}$ BW oligomer chitosan (5OC, 10OC), 5 or $10 \mathrm{mg}$ BW low-molecular-weight chitosan (5LMC, 10 LMC), 5 or $10 \mathrm{mg} / \mathrm{kg}$ BW high-molecularweight chitosan (5HMC, 10HMC) or $2.5 \mathrm{mg} / \mathrm{kg} \mathrm{BW}$ lovastatin (LOVA). Chitosan was administered four times per week for 12 weeks. Values are presented as the means $\pm \mathrm{SD}, \mathrm{n}=5-6$.

analysis. Following drying, $5 \mathrm{~g}$ of the feces was weighed for crude fat determination using the Soxhlet extractor method (24). Briefly, fecal samples in Soxhlet apparatus were exhaustively extracted by petroleum ether (boiling point range $40-60^{\circ} \mathrm{C}$ ) and allowed to run for $2 \mathrm{~h}$ to facilitate at least five cycles of Soxhlet extraction. The extracts were then evaporated to dryness over a steam bath and stored overnight in a desiccator. The added dry weight of the receptor-bottle was the net weight of total crude fat in each fecal sample.

SDS-polyacrylamide gel electrophoresis and immunoblotting. Liver and thoracic aorta tissues were homogenized in four volumes of a buffer ( $\mathrm{pH} 7.4)$ containing $10 \mathrm{mmol} / \mathrm{l}$ potassium phosphate, $150 \mathrm{mmol} / \mathrm{l}$ potassium chloride and $1 \mathrm{mM}$ phenylmethylsulfonyl fluoride, and centrifuged at $10,000 \times \mathrm{g}$ for $30 \mathrm{~min}$ at $4^{\circ} \mathrm{C}$. The supernatant was further ultracentrifuged at $105,000 \mathrm{x}$ g for $1 \mathrm{~h}$, and the final cytosolic supernatant was stored at $-80^{\circ} \mathrm{C}$ until analysis. Equal amounts of liver cytosolic fractions of each sample were applied to $10 \%$ SDS-polyacrylamide gels for $\mathrm{HMG}$ Co A reductase and ACAT-2 expression assay. Cytosolic fractions from the thoracic aorta were used for VCAM-1 and ICAM-1 protein analysis. Following electrophoresis, proteins separated on gels were transferred to polyvinylidine difluoride membranes and immunostained as described by Towbin et al (25). The membranes were incubated with $50 \mathrm{~g} / \mathrm{l}$ non-fat dry milk in

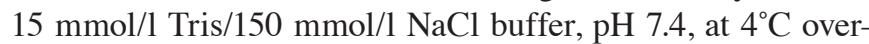
night to block non-specific binding. The membranes were then incubated with anti-HMG CoA reductase, ACAT-2, ICAM-1 or VCAM-1 antibody at $37^{\circ} \mathrm{C}$ for $1 \mathrm{~h}$, followed by peroxidase-conjugated secondary antibody. The bands were visualized using hydrogen peroxide/tetrahydrochloride diaminobenzidine or an enhanced chemiluminescent detection kit (Amersham Life Science, Buckinghamshire, UK) and were quantified with an AlphaImager 2000 (Alpha Innotech, San Leandro, CA, USA).
Additional biochemical assays. To examine whether the oral administration of chitosan affects liver function or caused liver toxicity, serum glutamic oxalacetic transaminase (GOT) and glutamate pyruvate transaminase (GPT) activities were determined using commercial assay kits (Randox Laboratories Ltd., San Diego, CA, USA) according to the manufacturer's instructions.

Statistical analysis. Data are expressed as the means \pm SD. Statistical analysis was performed using ANOVA (SAS Institute Inc., Cary, NC, USA). Tukey's multiple comparison was used to determine the significant difference among the group means $(\mathrm{P}<0.05)$.

\section{Results}

Animal characteristics. Following 12 weeks of feeding, changes in body weight, food intake and the relative visceral weight were determined. Compared to animals fed the 5\% soybean oil diet (ND), a HFD (20\% soybean oil) caused no changes in food intake, body weight gain nor an increase in the weight percentage of liver, heart, kidney and spleen to body weight. When hamsters were co-administered the three chitosans with either 5 or $10 \mathrm{mg} / \mathrm{kg}$, no effect on food intake, growth performance or the relative weight of the liver, spleen, heart, and kidney was noted (Table II). Moreover, hamsters administered chitosan showed no changes in serum GOT and GPT activities, suggesting that the chitosan dosage used caused no liver toxicity (Table III).

Blood lipid profile. Hamsters fed the HFD exhibited significantly higher serum TG, TC, VLDL-C and LDL-C levels than those noted in the low-fat ND group (Table IV). Upon co-treatment with chitosan and LOVA, three chitosan products resulted in lower serum TG (23-56\%), TC (19-48\%), VLDL-C (23-25\%) and LDL-C (24-44\%) in a dose-dependent 
Table III. Effects of different types of chitosan on the GOT and GPT in Syrian hamsters fed various diets for 12 weeks.

\begin{tabular}{lcc}
\hline Diet & GOT (units) & GPT (units) \\
\hline ND & $55 \pm 6$ & $77 \pm 10$ \\
HFD & $50 \pm 5$ & $75 \pm 11$ \\
HFD+5OC & $54 \pm 7$ & $68 \pm 11$ \\
HFD+10OC & $53 \pm 6$ & $72 \pm 11$ \\
HFD+5LMC & $52 \pm 6$ & $68 \pm 12$ \\
HFD+10LMC & $50 \pm 4$ & $73 \pm 14$ \\
HFD+5HMC & $51 \pm 5$ & $72 \pm 10$ \\
HFD+10HMC & $53 \pm 7$ & $67 \pm 13$ \\
HFD+LOVA & $55 \pm 7$ & $63 \pm 12$ \\
\hline
\end{tabular}

Hamsters were fed either a normal diet (ND) or high-fat diet (HFD) for 12 weeks. Animals fed the HFD were subgrouped and orally administered 5 or $10 \mathrm{mg} / \mathrm{kg} 6 \mathrm{kDa}$ oligomer chitosan (5OC, 10OC), 5 or $10 \mathrm{mg} / \mathrm{kg} 70 \mathrm{kDa}$ chitosan $(5 \mathrm{LMC}, 10 \mathrm{LMC}), 5$ or $10 \mathrm{mg} / \mathrm{kg}$ $200 \mathrm{kDa}$ chitosan (5HMC, 10HMC) or $2.5 \mathrm{mg} / \mathrm{kg}$ lovastatin (LOVA) four times per week. Values are presented as the means $\pm \mathrm{SD}, \mathrm{n}=5-6$.

manner compared with the HFD controls $(\mathrm{P}<0.05)$. The effectiveness of the three chitosans in lowering serum TG and cholesterol levels followed the order of $\mathrm{LMC}>\mathrm{OC}>\mathrm{HMC}$. Animals administered LOVA exhibited a lower level of TG, TC, VLDL-C and LDL-C than the HFD group $(\mathrm{P}<0.05)$. Serum contents of HDL-C were not affected by treatment with chitosan and LOVA.

Histological examination. A histological examination was performed to detect fatty liver changes. Feeding mice a HFD for 12 weeks (Fig. 1B) increased fatty liver. When co-administered with 5 or $10 \mathrm{mg} / \mathrm{kg}$ OC (Fig. 1C and D), LMC (Fig. 1E and F), or HMC (Fig. $1 \mathrm{G}$ and $\mathrm{H}$ ), fatty liver changes were suppressed. However, LOVA (Fig. 1I) offered minor protection to the fatty changes in liver tissues.
Total fecal crude fat levels. The total fecal fat content was measured to determine whether chitosan lowered lipid TG and cholesterol levels by interfering with fat absorption in the gastrointestinal tract. As shown in Fig. 2, following 12 weeks of 5 or $10 \mathrm{mg} / \mathrm{kg}$ LMC treatment, a 15 and $21 \%$ higher fecal fat content was noted, respectively. This was higher than that in the HFD-fed controls $(\mathrm{P}<0.05)$. However, administration of OC, HMC and LOVA did not change the feces fat content.

Hepatic HMG CoA reductase and ACAT-2 protein expression. Hepatic ACAT-2 and HMG CoA reductase protein levels were determined by immunoblot assay. The results indicated that HFD produced a higher ACAT-2 expression than that noted in ND-fed animals, although the difference was insignificant (Fig. 3A). When hamsters were co-treated with 5 or $10 \mathrm{mg} /$ $\mathrm{kg}$ OC, LMC and HMC or $2.5 \mathrm{mg} / \mathrm{kg}$ LOVA, hepatic ACAT-2 content significantly decreased $(\mathrm{P}<0.05)$. Regarding hepatic HMG CoA reductase, its expression was enhanced in hamsters fed the HFD diet $(\mathrm{P}<0.05)$ (Fig. 3B). However, chitosan and LOVA produced no changes in the hepatic content of this ratelimiting enzyme responsible for cholesterol synthesis.

VCAM-1 and ICAM-1 expression in thoracic aorta. Hyperlipidemia is associated with chronic inflammation in the vessel walls. Due to the effectiveness of chitosan at lowering blood lipids, this study examined the effect of chitosan suppression on the expression of ICAM-1 and VCAM-1, two adhesion molecules that are highly induced by inflammatory stimuli in the thoracic aorta. Unlike the ND-fed group, the HFD-fed animals exhibited much more VCAM-1 protein $(\mathrm{P}<0.05)$ (Fig. 4A). However, LMC, HMC, OC or LOVA treatment significantly suppressed HFD-induced VCAM-1 expression $(\mathrm{P}<0.05)$. Among the different types of chitosan, LMC and OC displayed greater potency than HMC $(\mathrm{P}<0.05)$. Regarding the changes in ICAM-1 in the thoracic aorta, a slight increase was produced by HFD feeding (Fig. 4B), but its expression was significantly decreased in hamsters treated with 5 and $10 \mathrm{mg} / \mathrm{kg} \mathrm{LMC}$ and $5 \mathrm{mg} / \mathrm{kg}$ OC $(\mathrm{P}<0.05)$. Note that HMC and LOVA did not change the ICAM-1 level.

Table IV. Effects of chitosan on blood lipids.

\begin{tabular}{|c|c|c|c|c|c|}
\hline Diet & TG & $\mathrm{TC}$ & LDL-C & HDL-C & VLDL-C \\
\hline ND & $144 \pm 10^{c}$ & $196 \pm 22^{\mathrm{d}}$ & $153 \pm 16^{\mathrm{b}, \mathrm{c}, \mathrm{d}}$ & $72 \pm 11$ & $28.7 \pm 2.0^{c}$ \\
\hline HFD & $282 \pm 19^{a}$ & $324 \pm 13^{a}$ & $236 \pm 15^{a}$ & $79 \pm 6$ & $56.5 \pm 3.8^{\mathrm{a}}$ \\
\hline $\mathrm{HFD}+5 \mathrm{OC}$ & $165 \pm 13^{b, c}$ & $198 \pm 20^{\mathrm{c}, \mathrm{d}}$ & $174 \pm 8^{\mathrm{b}, \mathrm{c}}$ & $76 \pm 8$ & $33.1 \pm 2.6^{b, c}$ \\
\hline HFD+10OC & $150 \pm 10^{c}$ & $205 \pm 21^{\mathrm{c}, \mathrm{d}}$ & $169 \pm 9^{\mathrm{b}, \mathrm{c}, \mathrm{d}}$ & $74 \pm 8$ & $30.1 \pm 1.9^{c}$ \\
\hline $\mathrm{HFD}+5 \mathrm{LMC}$ & $143 \pm 20^{c}$ & $181 \pm 12^{\mathrm{d}}$ & $138 \pm 15^{\mathrm{c}, \mathrm{d}}$ & $71 \pm 15$ & $28.7 \pm 3.9^{c}$ \\
\hline HFD+10LMC & $125 \pm 11^{\mathrm{c}}$ & $168 \pm 12^{\mathrm{d}}$ & $132 \pm 15^{\mathrm{d}}$ & $74 \pm 9$ & $25.1 \pm 2.3^{\mathrm{c}}$ \\
\hline HFD+5HMC & $216 \pm 31^{b}$ & $262 \pm 27^{b}$ & $180 \pm 12^{b}$ & $73 \pm 4$ & $43.2 \pm 6.1^{\mathrm{b}}$ \\
\hline HFD+10HMC & $180 \pm 21^{b, c}$ & $249 \pm 18^{b, c}$ & $174 \pm 13^{\mathrm{b}, \mathrm{c}}$ & $75 \pm 6$ & $35.9 \pm 4.3^{b, c}$ \\
\hline HFD+LOVA & $216 \pm 25^{b}$ & $161 \pm 15^{\mathrm{d}}$ & $130 \pm 18^{\mathrm{d}}$ & $73 \pm 6$ & $43.2 \pm 5.1^{\mathrm{b}}$ \\
\hline
\end{tabular}

Hamsters were fed either a normal diet (ND) or high-fat diet (HFD) for 12 weeks. Animals fed the HFD were orally administered 5 or $10 \mathrm{mg} /$ $\mathrm{kg}$ oligomer chitosan (5OC, 10OC), 5 or $10 \mathrm{mg} / \mathrm{kg}$ low-molecular-weight chitosan (5LMC, $10 \mathrm{LMC}$ ), 5 or $10 \mathrm{mg} / \mathrm{kg}$ high-molecular-weight chitosan (5HMC, 10HMC) or $2.5 \mathrm{mg} / \mathrm{kg} \mathrm{BW}$ lovastatin (LOVA) four times per week. Serum total triglycerides (TG), total cholesterol (TC), LDL-cholesterol (LDL-C), HDL-cholesterol (HDL-C) and VLDL-cholesterol (VLDL-C) were determined. Values are presented as the means $\pm \mathrm{SD}, \mathrm{n}=5-6{ }^{\mathrm{a}, \mathrm{b}, \mathrm{c}, \mathrm{d}}$ Group mean values not sharing the same symbols differed significantly, $\mathrm{P}<0.05$. Blood lipid values are expressed as $\mathrm{mg} / \mathrm{dl}$. 

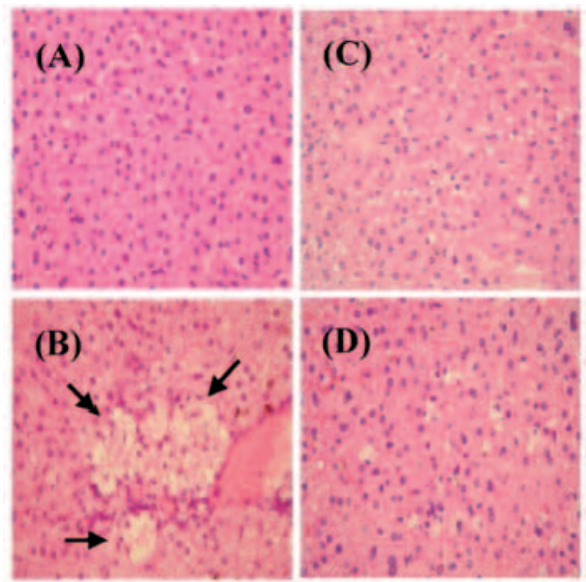
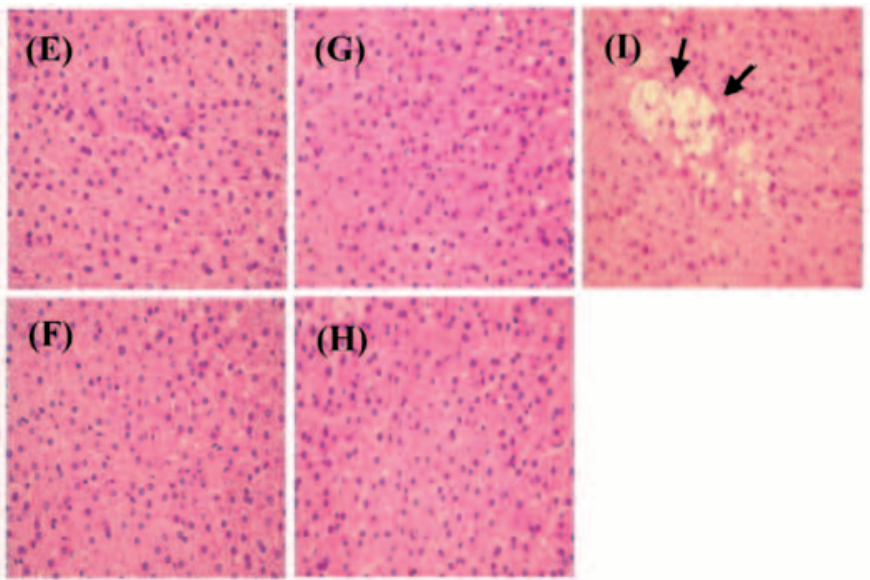

Figure 1. Fatty liver formation in Syrian hamsters fed a high-fat diet and chitosan of different molecular weights. Animals were fed (A) normal or (B) high-fat diets for 12 weeks. Hamsters in the high-fat diet were further subgrouped and orally administered (C and D) oligomer chitosan, (E and F) 5 or $10 \mathrm{mg} / \mathrm{kg}$ low-molecular-weight chitosan, (G and H) high-molecular-weight chitosan, or (I) $2.5 \mathrm{mg} / \mathrm{kg}$ lovastatin four times per week. Tissue sections were stained with hematoxylin and eosin (magnification, x200). The typical fatty changes are indicated by arrows.

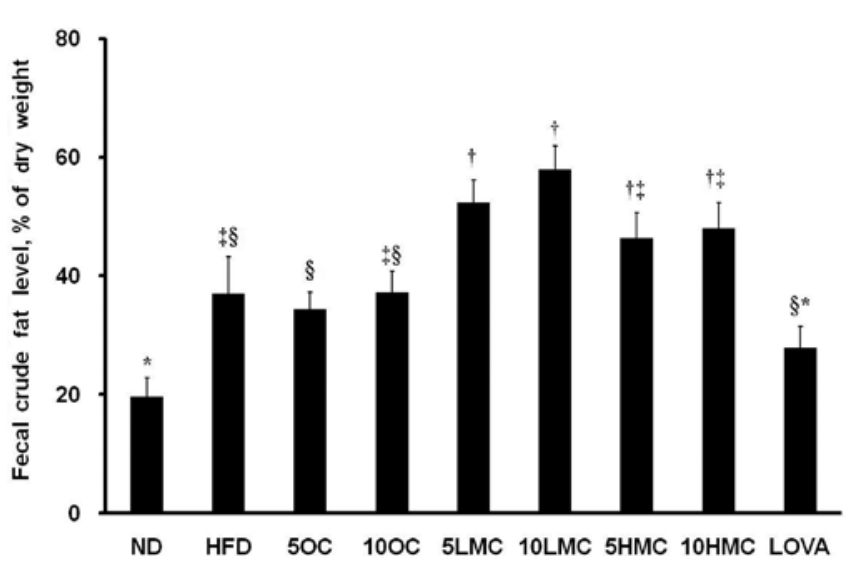

Figure 2. Changes in the fecal fat content by chitosans. Hamsters were fed either a low-fat diet (ND) or high-fat diet (HFD). Animals fed the HFD were sub-grouped and orally administered 5 or $10 \mathrm{mg} / \mathrm{kg}$ oligo-form chitosan (5OC or 10OC), low-molecular-weight chitosan (5LMC or 10LMC), high-molecular-weight chitosan (5HMC or $10 \mathrm{HMC}$ ), or $2.5 \mathrm{mg} / \mathrm{kg}$ lovastatin (LOVA) for 12 weeks. Feces were collected during the week prior to animal sacrifice, and the crude feces fat content was determined as described in Materials and methods. Values are presented as the means $\pm S D, n=5-6$. ${ }_{\dagger}^{\dagger} \mathrm{s}^{*}$ Values not sharing the same letters differed significantly, $\mathrm{P}<0.05$.

\section{Discussion}

Recent studies indicate that chitosan is effective at decreasing food lipid and cholesterol absorption in the gastrointestinal tract. As a result, chitosan is a functional anti-obesity food, and is capable of lowering blood triglycerides and cholesterol $(3,10,26)$. Chitosan, a biopolymer of glucosamine, is produced by the deacetylation of chitin and consists of polymers with various molecular weights. The hypolipidemic activity of chitosan is related to a number of chemical characteristics, including molecular weight, degree of acetylation and viscosity $(6,27)$. Regarding the effect of the differential molecular weight, feeding an obese mouse model a HFD showed that chitosan with $46 \mathrm{kDa}$ molecular weight is more effective at attenuating body weight gain and lowering blood total triglycerides and total cholesterol and LDL-cholesterol

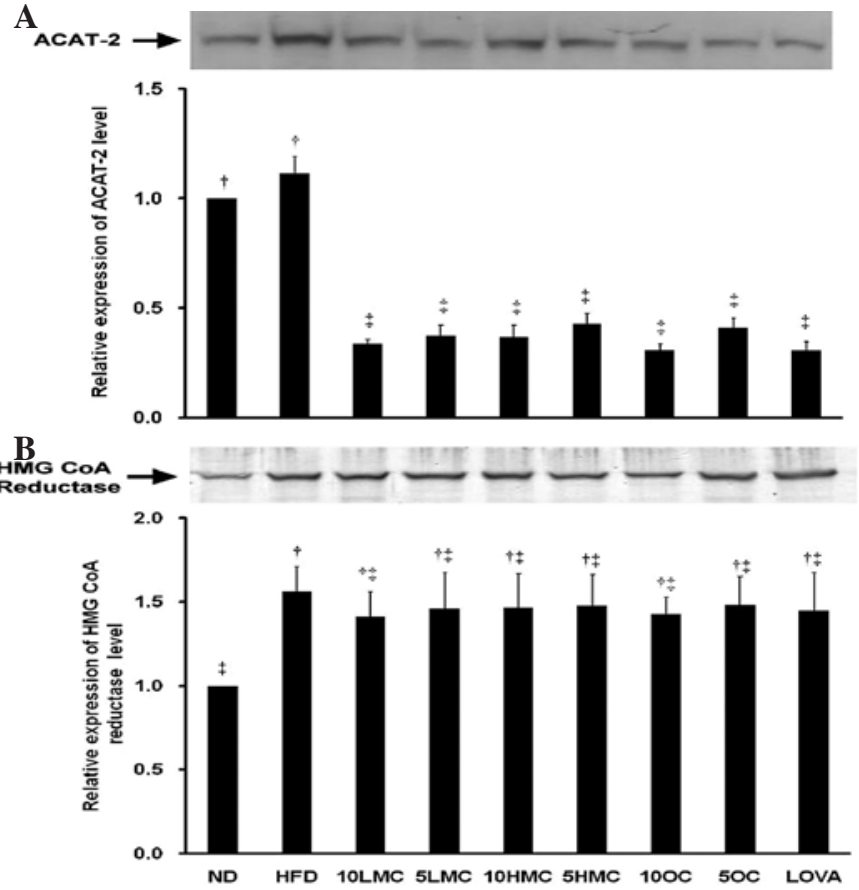

Figure 3. Hepatic HMG CoA reductase and ACAT-2 protein expression in hamsters treated with various chitosans. Following 12 weeks of feeding ND or HFD or HFD plus 5 or $10 \mathrm{mg} / \mathrm{kg} \mathrm{OC,} \mathrm{LMC,} \mathrm{or} \mathrm{HMC,} \mathrm{or} 2.5 \mathrm{mg} / \mathrm{kg}$ lovastatin (LOVA), (A) HMG CoA reductase and (B) ACAT-2 expression in liver tissues was measured by immunoblotting assay. HMG CoA reductase and ACAT-2 expression in the ND group was treated as 1. Values are presented

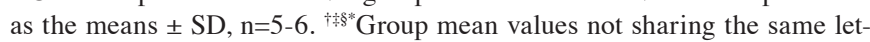
ters differed significantly, $\mathrm{P}<0.05$.

than $650 \mathrm{kDa}$ chatoyant (6). This finding agrees with the current study, which shows that lower molecular weight chitosan $(70 \mathrm{kDa})$ displays better hypolipidemic and hypocholesterolemic activity than $200 \mathrm{kDa}$ chitosan (Table IV). Moreover, low-molecular-weight chitosan results in a higher lipid contents in feces than the high-molecular-weight product (Fig. 2). The increase in fecal fat content caused by chitosan is attributed to the formation of agglomerates with fatty acids 


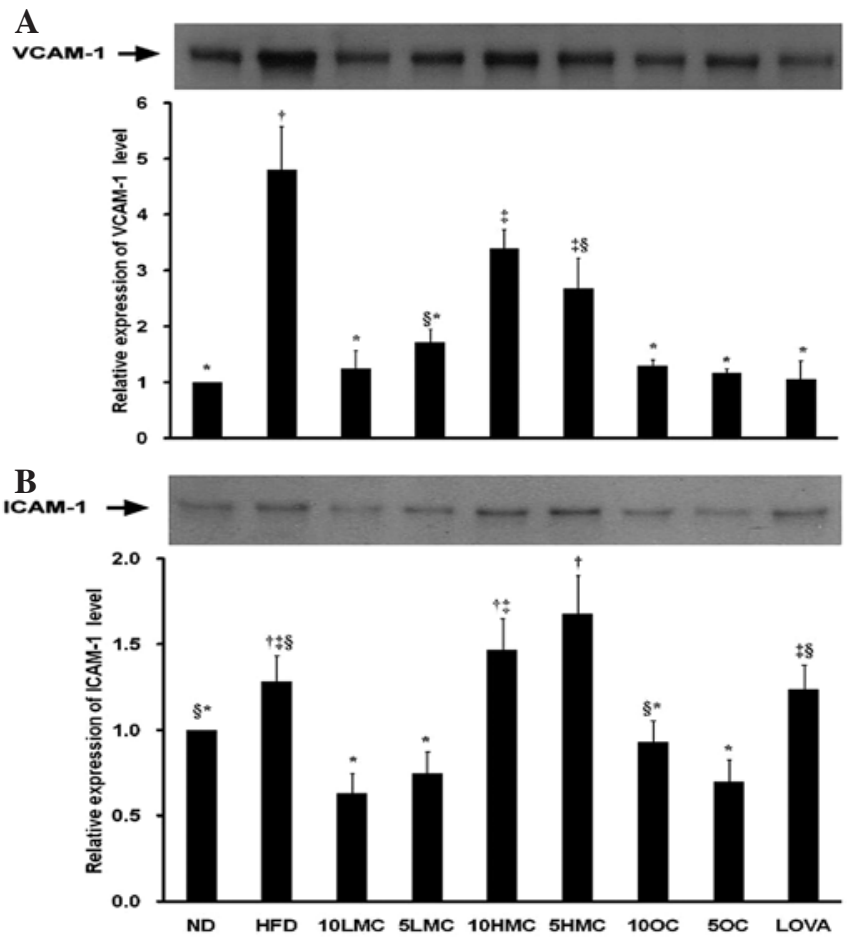

Figure 4. Effects of chitosan on thoracic aortic VCAM-1 and ICAM-1 protein expression. Hamsters were fed a diet with 5\% soybean oil (ND) or $20 \%$ soybean oil $/ 0.5 \%$ cholesterol (HFD). Animals fed the HFD were subgrouped and orally administered 5 or $10 \mathrm{mg} / \mathrm{kg} \mathrm{OC}, \mathrm{LMC}$ or $\mathrm{HMC}$, or $2.5 \mathrm{mg} / \mathrm{kg}$ lovastatin (LOVA) for 12 weeks. Thoracic aortic (A) VCAM-1 and (B) ICAM-1 protein levels were measured by immunoblotting assay. VCAM-1 and ICAM-1 expression in the ND group was treated as 1 . Values are presented as the means $\pm \mathrm{SD}, \mathrm{n}=5-6$. ${ }^{\dagger}+8^{*}$ Group mean values not sharing the same letters differed significantly, $\mathrm{P}<0.05$.

and cholesterol in the gastrointestinal tract $(12,28)$. These findings suggest that the differential potency on the decrease in dietary lipid absorption explains, at least partly, the difference in the blood lipid lowering effect of $70 \mathrm{kDa}$ and $200 \mathrm{kDa}$ chatoyant (12,28-30). In addition, the chitosan decrease of fatty liver (Table IV) suggests that glucosamine polymers may affect lipid metabolism in the liver.

To determine the optimal range of chitosan molecular weight on hypolipidemia, this study also tested an oligo-form chitosan of $6 \mathrm{kDa}$. Recent in vitro experiments indicate that the oligo-form of chitosan displays anti-tumorigenesis, anti-adipogenesis and apoptosis activity. For instance, 1-3 kDa chitosan was found to inhibit the inflammatory response in HT-29 cells induced by cytokines (31) and to suppress adipogenesis of 3T3-L1 preadipocytes (32). In addition, 3-5 kDa oligoform chitosan inhibited colon cancer formation by increasing quinone reductase and glutathione S-transferase activities and glutathione levels, and inhibited ornithine decarboxylase activity and cyclooxygenase-2 expression in HT-29 cells (33). However, the results of this study clearly indicate that the effects of $6 \mathrm{kDa}$ oilgo-form chitosan on lowering blood lipids (Table IV) and inhibiting intestinal fat absorption (Fig. 2) are inferior to $70 \mathrm{kDa}$ chitosan. These results suggest that, in an in vivo model, chitosan with an optimal molecular weight is required for approaching the best blood lowering activity.

ACAT-2 plays a significant role in hepatic cholesterol metabolism $(20,21)$. In the liver, ACAT catalyzes the intra- cellular cholesteryl ester production from esterification of cholesterol and is involved in the production and release of apoB-containing lipoproteins (34). Higher ACAT activity speeds up the release of VLDL from the liver, leading to higher blood cholesterol levels. The hypocholesterolemic effect of a number of phytochemicals has been attributed to their action on inhibiting ACAT activity and expression. For instance, the effects of pinitol and naringenin on lowering total blood cholesterol and LDL-cholesterol levels in hamsters and rats fed a HFD is explained partly by their effectiveness at down-regulating ACAT expression in liver tissue $(35,36)$. Bavachin and isobavachalcone are potent ACAT inhibitors in HepG2 cells (23). This study shows that in addition to lowering blood VLDL- and LDL-cholesterol levels, either low- or highmolecular-weight chitosan or oligomer chitosan significantly decreased hepatic ACAT-2 expression in hamsters (Fig. 3A). By contrast, the hypocholesterolemic effect of chitosan is likely to be independent of the modulation of $\mathrm{HMG} \mathrm{CoA}$ reductase expression (Fig. 3B).

In the early stage of atherosclerosis, endothelial cells express a variety of cellular adhesion molecules, such as VCAM-1 and ICAM-1. These molecules play a significant role in recruiting circulating leukocytes to the vascular endothelium and facilitating their penetration into the vascular intima (37). The interruption of the abnormal induction of adhesion molecules under certain circumstances, such as chronic inflammation, hypercholesterolemia and oxidative stress, ameliorates the progression of this blood vessel disease (17,38). The expression of ICAM-1 and VCAM-1 in endothelial cells is highly inducible under certain stimuli, including proinflammatory cytokines and oxidative stress. Higher ICAM-1 and VCAM-1 levels are associated with hypercholesterolemia $(39,40)$. Oxidized LDL stimulates endothelial cells to produce MCP-1, ICAM-1, VCAM-1 and E- and $\mathrm{P}$-selectin and increases monocyte recruitment and infiltration into the lesions of the endothelium. The suppression of abnormal adhesion molecule expression in vascular endothelium is thought to protect against the incidence of atherosclerosis $(41,42)$. It is interesting to examine whether chitosan decreases adhesion molecule expression. This study revealed a 4-fold increase in the VCAM-1 level in the thoracic aorta of hamsters fed the HFD compared to the ND diet (Fig. 4A). After co-administration with chitosan, VCAM-1 expression was significantly suppressed, with $70 \mathrm{kDa}$ and $7 \mathrm{kDa}$ chitosan displaying higher inhibitory potency than that of $200 \mathrm{kDa}$ high-molecular-weight chitosan. A similar inhibition on HFD-induced ICAM-1 expression was also noted by dosing with $70 \mathrm{kDa}$ and $7 \mathrm{kDa}$ chitosan (Fig. 4B). The similar pattern of decreasing blood lipid levels and vascular VCAM-1 and ICAM-1 expression among the three chitosans of different molecular weight suggests that the hypolipidemic effect of chitosan is through the amelioration of chronic inflammation and oxidative stress, which in turn attenuates the adhesion molecules induced by a HFD $(43,44)$.

In conclusion, the results of this study indicate that chitosan effectively lowers blood triglycerides, total cholesterol and VLDL- and LDL-cholesterol and further suppresses vascular adhesion molecule expression in hamsters fed a HFD. This effect is partly attributable to the chitosan inhibition of intestinal lipid absorption and hepatic ACAT-2 
expression. Moreover, the hypolipidemic potency depends on the molecular weight of chitosan.

\section{Acknowledgements}

This study was supported by Grants FY95-RD-003 from Fooyin University and Taiwan Hopax Chemicals Mfg. Co., Ltd., Kaohsiung, Taiwan, and NSC 96-2320-B-309-005-MY3 from the National Science Council, Taiwan.

\section{References}

1. Lee E, Kim H, Lee IH and Jon S: In vivo antitumor effects of chitosan-conjugated docetaxel after oral administration. J Control Release 140: 79-85, 2009.

2. Yuan WP, Liu B, Liu CH, et al: Antioxidant activity of chito-oligosaccharides on pancreatic islet cells in streptozotocin-induced diabetes in rats. World J Gastroenterol 15: 1339-1345, 2009.

3. Chiang MT, Yao HT and Chen HC: Effect of dietary chitosans with different viscosity on plasma lipids and lipid peroxidation in rats fed on a diet enriched with cholesterol. Biosci Biotechnol Biochem 64: 965-971, 2000.

4. Mhurchu CN, Poppitt SD, McGill AT, et al: The effect of the dietary supplement, chitosan, on body weight: a randomised controlled trial in 250 overweight and obese adults. Int J Obes Relat Metab Disord 28: 1149-1156, 2004

5. No HK, Park NY, Lee SH and Meyers SP: Antibacterial activity of chitosans and chitosan oligomers with different molecular weights. Int J Food Microbiol 74: 65-72, 2002.

6. Sumiyoshi M and Kimura Y: Low molecular weight chitosan inhibits obesity induced by feeding a high-fat diet long-term in mice. J Pharm Pharmacol 58: 201-207, 2006.

7. Rodriguez MS and Albertengo LE: Interaction between chitosan and oil under stomach and duodenal digestive chemical conditions. Biosci Biotechnol Biochem 69: 2057-2062, 2005.

8. Gallaher DD, Gallaher CM, Mahrt GJ, et al: A glucomannan and chitosan fiber supplement decreases plasma cholesterol and increases cholesterol excretion in overweight normocholesterolemic humans. J Am Coll Nutr 21: 428-433, 2002.

9. Qin C, Du Y, Xiao L, Li Z and Gao X: Enzymic preparation of water-soluble chitosan and their antitumor activity. Int J Bio Macromol 31: 111-117, 2002.

10. Razdan A and Pettersson D: Effect of chitin and chitosan on nutrient digestibility and plasma lipid concentrations in broiler chickens. Br J Nutr 72: 277-288, 1994

11. LeHoux JG and Grondin F: Some effects of chitosan on liver function in the rat. Endocrinology 132: 1078-1084, 1993.

12. Sugano M, Fujikawa T, Hiratsuji Y, Nakashima K, Fukuda N and Hasegawa Y: A novel use of chitosan as a hypocholesterolemic agent in rats. Am J Clin Nutr 33: 787-793, 1980.

13. Mun S, Decker EA and McClements DJ: Effect of molecular weight and degree of deacetylation of chitosan on the formation of oil-in-water emulsions stabilized by surfactant-chitosan membranes. J Colloid Interface Sci 296: 581-590, 2006.

14. Pervaiz SM and Abdul Haleem M: Studies on the structure of beta-chitin, I. Z Naturforsch C 30: 571-574, 1975.

15. Luskey KL: Regulation of cholesterol synthesis: mechanism for control of HMG CoA reductase. Recent Prog Horm Res 44 35-51, 1988.

16. Goldstein JL and Brown MS: Regulation of the mevalonate pathway. Nature 343: 425-430, 1990.

17. Lei YP, Chen HW, Sheen LY and Lii CK: Diallyl disulfide and diallyl trisulfide suppress oxidized LDL-induced vascular cell adhesion molecule and E-selectin expression through protein kinase A- and B-dependent signaling pathways. J Nutr 138 996-1003, 2008.

18. Sung JH, Lee SJ, Park KH and Moon TW: Isoflavones inhibit 3-hydroxy-3-methylglutaryl coenzyme A reductase in vitro. Biosci Biotechnol Biochem 68: 428-432, 2004.

19. Liu L and Yeh YY: S-alk(en)yl cysteines of garlic inhibit cholesterol synthesis by deactivating HMG-CoA reductase in cultured rat hepatocytes. J Nutr 132: 1129-1134, 2002.

20. Chang TY, Chang CC, Lin S, Yu C, Li BL and Miyazaki A: Roles of acyl-coenzyme A:cholesterol acyltransferase-1 and -2 . Curr Opin Lipidol 12: 289-296, 2001.
21. Chang TY, Chang CC and Cheng D: Acyl-coenzyme A:cholesterol acyltransferase. Annu Rev Biochem 66: 613-638, 1997.

22. Miyazaki A, Sakashita N, Lee O, et al: Expression of ACAT-1 protein in human atherosclerotic lesions and cultured human monocytes-macrophages. Arterioscler Thromb Vasc Biol 18: 1568-1574, 1998.

23. Choi JH, Rho MC, Lee SW, et al: Bavachin and isobavachalcone, acyl-coenzyme A: cholesterol acyltransferase inhibitors from Psoralea corylifolia. Arch Pharm Res 31: 1419-1423, 2008.

24. Han C, Chen X, Xie W, et al: Determination of hexabromocyclododecane diastereoisomers in Sargassum fusiforme and comparison of the extraction efficiency of ultrasonication, microwave-assisted extraction, Soxhlet extraction and pressurised liquid extraction. J Sep Sci 33: 3319-3325, 2010.

25. Towbin H, Staehelin T and Gordon J: Electrophoretic transfer of proteins from polyacrylamide gels to nitrocellulose sheets: procedure and some applications. Proc Natl Acad Sci USA 76: 4350-4354, 1979.

26. Zhang J, Liu J, Li L and Xia W: Dietary chitosan improves hypercholesterolemia in rats fed high-fat diets. Nutr Res 28: 383-390, 2008.

27. Razdan A and Pettersson D: Hypolipidaemic, gastrointestinal and related responses of broiler chickens to chitosans of different viscosity. Br J Nutr 76: 387-397, 1996.

28. Ebihara K and Schneeman BO: Interaction of bile acids, phospholipids, cholesterol and triglyceride with dietary fibers in the small intestine of rats. J Nutr 119: 1100-1106, 1989.

29. Nestel PJ: Effects of dairy fats within different foods on plasma lipids. J Am Coll Nutr 27: 735S-740S, 2008.

30. Katcher HI, Hill AM, Lanford JL, Yoo JS and Kris-Etherton PM: Lifestyle approaches and dietary strategies to lower LDL-cholesterol and triglycerides and raise HDL-cholesterol. Endocrinol Metab Clin North Am 38: 45-78, 2009.

31. Nam KS, Kim MK and Shon YH: Inhibition of proinflammatory cytokine-induced invasiveness of HT-29 cells by chitosan oligosaccharide. J Microbiol Biotechnol 17: 2042-2045, 2007.

32. Cho EJ, Rahman MA, Kim SW, et al: Chitosan oligosaccharides inhibit adipogenesis in 3T3-L1 adipocytes. J Microbiol Biotechnol 18: 80-87, 2008.

33. Nam KS, Kim MK and Shon YH: Chemopreventive effect of chitosan oligosaccharide against colon carcinogenesis. J Microbiol Biotechnol 17: 1546-1549, 2007.

34. Katsuren K, Tamura T, Arashiro R, et al: Structure of the human acyl-CoA:cholesterol acyltransferase-2 (ACAT-2) gene and its relation to dyslipidemia. Biochim Biophys Acta 1531: 230-240, 2001.

35. Jeon SM, Kim HK, Kim HJ, et al: Hypocholesterolemic and antioxidative effects of naringenin and its two metabolites in high-cholesterol fed rats. Transl Res 149: 15-21, 2007.

36. Choi MS, Lee MK, Jung UJ, et al: Metabolic response of soy pinitol on lipid-lowering, antioxidant and hepatoprotective action in hamsters fed-high fat and high cholesterol diet. Mol Nutr Food Res 53: 751-759, 2009.

37. Galkina E and Ley K: Vascular adhesion molecules in atherosclerosis. Arterioscler Thromb Vasc Biol 27: 2292-2301, 2007.

38. Stangl V, Lorenz M, Ludwig A, et al: The flavonoid phloretin suppresses stimulated expression of endothelial adhesion molecules and reduces activation of human platelets. J Nutr 135: $172-178,2005$.

39. Gourdin MJ, Bree B and De Kock M: The impact of ischaemiareperfusion on the blood vessel. Eur J Anaesthesiol 26: 537-547, 2009.

40. Bacun T, Glavas-Obrovac L, Belovari T, et al: Insulin administration in the mild hyperglycaemia changes expression of proinflammatory adhesion molecules on human aortic endothelial cells. Coll Antropol 34: 911-915, 2010.

41. Kita T, Kume N, Minami M, et al: Role of oxidized LDL in atherosclerosis. Ann NY Acad Sci 947: 199-206, 2001.

42. Kita T: Hyperlipidemia and atherosclerosis. Nihon Rinsho 60: 851-859, 2002.

43. Fang W, Zhang HX, Wang LY, et al: The effects of polygoni multiflori total glycosides on the experimentally atherosclerotic formation in apoE-deficient mice. Zhongguo Zhong Yao Za Zhi 30: 1542-1545, 2005.

44. Fang W, Zhang HX, Wang LY, et al: Effect of PMTG on atherosclerotic lesion formation and expression of ICAM-1 and VCAM-1 in ApoE-deficient mice. Zhongguo Zhong Yao Za Zhi 32: 1320-1323, 2007. 